\title{
Activación de char de lodo de EDAR y de purín de vacuno mediante oxidación
}

\author{
María Atienza-Martínez ${ }^{1}$, Nurull Nadia Binti Suraini ${ }^{2}$, Héctor Bautista ${ }^{2}$, Javier \\ Ábrego ${ }^{1}$, Gloria Gea ${ }^{1}$ \\ ${ }^{1}$ GPT-Grupo de Procesos Termoquímicos \\ Instituto de Investigación en Ingeniería de Aragón (I3A) \\ Universidad de Zaragoza, Mariano Esquillor s/n, 50018, Zaragoza, Spain. \\ Tel. +34-976762707, e-mail: atienza@unizar.es \\ ${ }^{2}$ Departamento de Ingeniería Química y Tecnologías del Medio Ambiente, Universidad de Zaragoza
}

\section{Resumen}

En este trabajo se plantea la activación mediante oxidación del producto sólido (char) que se obtiene en la pirólisis de la fracción sólida digerida de lodo de estaciones depuradoras de aguas residuales (EDAR) y de purín de vacuno.

\section{Introducción y objetivos}

La digestión anaerobia es una de las tecnologías más empleadas para el tratamiento de residuos orgánicos como el lodo de las estaciones depuradoras de aguas residuales (EDAR) o los purines de animales. En este proceso, además de una fracción sólida digerida (FSD), se genera una corriente residual líquida (fracción líquida digerida, FLD) que habitualmente tiene una elevada concentración de amonio $\left(\mathrm{NH}_{4}^{+}\right)$. Para poder retornar esta FLD a la cabecera de las plantas de digestión anaerobia es necesario reducir su contenido en $\mathrm{NH}_{4}{ }^{+}$. La FSD puede ser empleada como fertilizante, pero también puede ser sometida a otros tratamientos como los procesos termoquímicos, entre los que podemos encontrar la pirólisis [1].

El producto sólido (char) que se obtiene en la pirólisis de la FSD puede emplearse para adsorber el $\mathrm{NH}_{4}{ }^{+}$presente en la FLD, lo que puede considerarse como una tecnología económica para eliminar este compuesto [2]. La retención de $\mathrm{NH}_{4}{ }^{+}$en el char está relacionada con la adsorción electrostática por medio de grupos funcionales superficiales que contienen oxígeno y que están cargados negativamente [3]. La oxidación del char con oxígeno puede incrementar la concentración de este tipo de grupos funcionales superficiales. Por lo tanto, la reducción de la concentración de $\mathrm{NH}_{4}{ }^{+}$en la FLD mediante char previamente activado con oxígeno puede integrarse en las plantas de tratamiento.
El objetivo de este trabajo es estudiar la oxidación del char de pirólisis de lodo de EDAR y de purín de vacuno, mediante análisis termogravimétrico, con el fin de aumentar en su superficie el contenido en grupos oxigenados lo que podría favorecer su uso como sólidos adsorbentes para la reducción de $\mathrm{NH}_{4}{ }^{+}$ en una corriente acuosa.

\section{Materiales y métodos}

\section{Materiales}

Los materiales que se han empleado han sido lodo de EDAR y purín de vacuno, ambos digeridos anaeróbicamente. Las propiedades de estos dos materiales se muestran en la Tabla 1.

\section{Métodos}

Los experimentos se han realizado en una termobalanza NETZSCH STA 449 F3 Jupiter. En una primera etapa, se ha llevado a cabo la pirólisis de lodo de EDAR o de purín de vacuno a $550{ }^{\circ} \mathrm{C}$ bajo atmósfera de $\mathrm{N}_{2}$. En la segunda etapa se ha realizado la activación del char obtenido en la primera etapa, a dos temperaturas de activación distintas $\left(200{ }^{\circ} \mathrm{C}\right.$ y $\left.350{ }^{\circ} \mathrm{C}\right)$, un tiempo de activación de 30 min y una concentración de oxígeno en el gas de $21 \%$ vol.

\section{Resultados}

Tanto en la Figura 1 (temperatura de activación de $200{ }^{\circ} \mathrm{C}$ ) como en la Figura 2 (temperatura de activación de $350{ }^{\circ} \mathrm{C}$ ) se observa como durante la etapa de pirólisis se pierde aproximadamente el 50\% de la masa inicial de lodo de EDAR y el $60 \%$ en el caso del purín de vacuno. El mayor grado de descomposición de este último se explica por su menor contenido en cenizas (ver Tabla 1). Cuando la activación se lleva a cabo a $200^{\circ} \mathrm{C}$, tanto en el caso del lodo de EDAR como en el del purín de 
vacuno se observa que al inicio de la etapa de activación la masa de muestra aumenta ligeramente (en torno a un $1 \%$ ) y posteriormente no se observa pérdida de masa con el tiempo. Este aumento de masa podría deberse a que parte del oxígeno presente en la atmósfera de reacción queda en la superficie del char. Cuando la etapa de activación se lleva a cabo bajo condiciones de operación más severas (temperatura de $350{ }^{\circ} \mathrm{C}$ ), la ganancia de masa al inicio de esta etapa es inferior al $0,5 \%$ y pronto se observa una nueva pérdida de masa. Al final de esta etapa, se ha perdido el $57 \%$ y el $63 \%$ del lodo de EDAR y el purín de vacuno, respectivamente. $\mathrm{El}$ análisis mediante espectroscopía de infrarrojos por transformada de Fourier (FTIR) de las muestras de char activado corroboran el posible aumento de los grupos oxigenados en la superficie del char cuando la activación se ha llevado a cabo bajo condiciones moderadas $\left(200{ }^{\circ} \mathrm{C}, 30 \mathrm{~min}\right.$ y concentración de oxígeno del $21 \%$ ).

\section{Conclusiones}

Con el objetivo de favorecer el aumento de la proporción de grupos funcionales oxigenados en la superficie del char de pirólisis de lodo de EDAR y de purín de vacuno interesa controlar las condiciones de operación a las que se lleva a cabo la

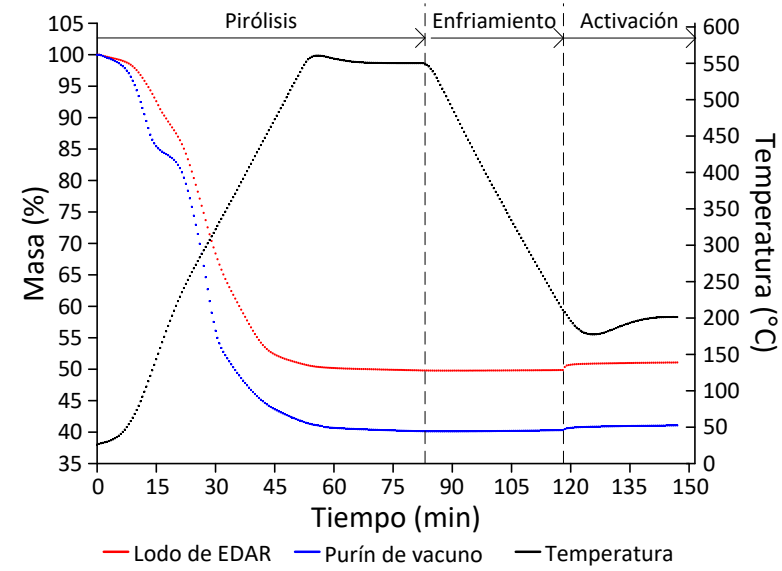

Fig 1. Evolución de la masa de sólido durante la pirólisis y la activación (temperatura de activación de $200{ }^{\circ} \mathrm{C}$ ). etapa de activación, para lograr que se incorpore el oxígeno a la superficie del char evitando que sean lo suficientemente severas como para que tenga lugar la combustión de gran parte de la materia orgánica presente en estos residuos.

\section{REFERENCIAS}

[1]. CZAJCZYŃSK, D., ANGUILANO, L., GHAZAL, H., KRZYŻYŃSKA, R., REYNOLDS, A.J., SPENCER, N., JOUHAR, H. Potential of Pyrolysis Processes in the Waste Management Sector. Thermal Science and Engineering Progress. 2017, 3, 171-197. Available from: doi: 10.1016/j.tsep.2017.06.003.

[2]. TAKAYA, C.A., FLETCHER, L.A., SINGH, S., ANYIKUDE, K.U., ROSS, A.B. Phosphate and ammonium sorption capacity of biochar and hydrochar from different wastes. Chemosphere. 2016, 145, 518-527. Available from: doi: 10.1016/j.chemosphere.2015.11.052.

[3]. HALE, S.E., ALLING, V., MARTINSEN, V., MULDER, J., BREEDVELD, G.D., CORNELISSEN, G. The sorption and desorption of phosphate- $\mathrm{P}$, ammonium- $\mathrm{N}$ and nitrate- $\mathrm{N}$ in cacao shell and corn cob biochars. Chemosphere. 2013 91(11), 1612-1619. Available from: doi: 10.1016/j.chemosphere.2012.12.057.

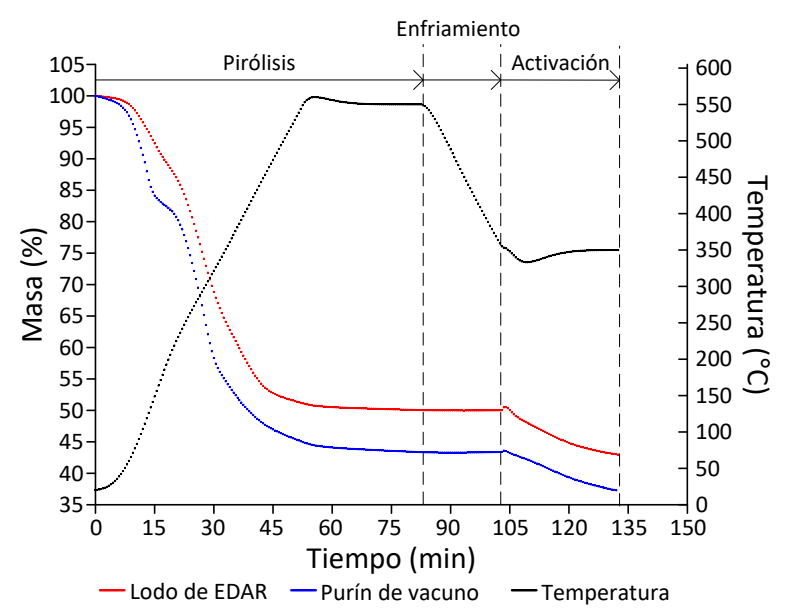

Fig 2. Evolución de la masa de sólido durante la pirólisis y la activación (temperatura de activación de $350{ }^{\circ} \mathrm{C}$ ).

Tabla 2. Caracterización de los materiales empleados.

\begin{tabular}{|l|l|l|l|l|l|l|l|l|l|}
\hline & $\begin{array}{l}\text { Humedad } \\
(\%)\end{array}$ & $\begin{array}{l}\text { Cenizas } \\
(\%)\end{array}$ & $\begin{array}{l}\text { Volátiles } \\
(\%)\end{array}$ & $\begin{array}{l}\text { Carbono } \\
\text { fijo }(\%)\end{array}$ & $\begin{array}{l}\mathrm{C} \\
(\%)\end{array}$ & $\begin{array}{l}\mathrm{H} \\
(\%)\end{array}$ & $\begin{array}{l}\mathrm{N} \\
(\%)\end{array}$ & $\begin{array}{l}\mathrm{S} \\
(\%)\end{array}$ & $\begin{array}{l}\mathrm{O} \\
(\%)\end{array}$ \\
\hline $\begin{array}{l}\text { Lodo } \\
\text { EDAR }\end{array}$ & 6,5 & 39,0 & 50,0 & 4,5 & 29,5 & 4,7 & 5,3 & 1,3 & 20,2 \\
\hline $\begin{array}{l}\text { Purín de } \\
\text { vacuno }\end{array}$ & 19,0 & 22,0 & 49,0 & 7,0 & 32,3 & 4,9 & 1,9 & 0,5 & 38,4 \\
\hline
\end{tabular}

